\title{
A Survey on Applications of Adaptive Neuro Fuzzy Inference System
}

\author{
Navneet Walia ${ }^{1}$, Sharad Kumar ${ }^{2}$ and Harsukhpreet Singh $^{3}$ \\ ${ }^{1,2}$ CT Institute of Technology and Research, Jalandhar \\ ${ }^{3}$ Thapar University, Patiala \\ navneetwalia356@gmail.com
}

\begin{abstract}
In this paper we presented an architecture and basic learning process underlying in fuzzy inference system and adaptive neuro fuzzy inference system which is a hybrid network implemented in framework of adaptive network. In real world computing environment, soft computing techniques including neural network, fuzzy logic algorithms have been widely used to derive an actual decision using given input or output data attributes, ANFIS can construct mapping based on both human knowledge and hybrid learning algorithms. This study involves study of ANFIS strategy ANFIS strategy is employed to model nonlinear functions, to control one of the most important parameters of the induction machine and predict a chaotic time series, all yielding more effective, faster response or settling times.
\end{abstract}

Keywords: ANFIS, Fuzzy logic, neural network, decision support system, learning algorithm

\section{Introduction}

Technological innovations in soft computing aim to exploit tolerance for imprecision and have brought automation capabilities to new levels of applications. Process control is an important application of any industry for controlling the complex system parameter and to provide low cost solution. Soft computing techniques can cope with variety of environmental and stability related uncertainties applications. These techniques consist of fuzzy logic (FL), neural network (NN) and genetic algorithms (GA) methodologies to design state-of-art intelligent systems ranging from computer aided diagnosis, computer aided recognition or in intensive care unit [1]. In methodically, the control techniques based on fuzzy modeling or fuzzy identification was first systematically introduced by Takagi and Sugeno, has found diverse applications in fuzzy control for medical diagnosis [3], decision-making and solve problems based on data mining. However, there are some fundamental aspects of this approach which are in need of better understanding. More specifically, the lack of standard design procedure and optimization process to transform human knowledge or experience into rule base and the data base of the fuzzy inference system [2]. In this paper, we describes a novel class of neuro-fuzzy architecture called Adaptive Neuro-Fuzzy Inference System (ANFIS) with ultimate aim to explain fuzzy inference system via learning and has been widely employed to represent or approximate a nonlinear system. Adaptive systems can be described by constructing a set of fuzzy ifthen rules that represent local linear input-output relations of the system. All these methodologies work together and provide flexible information capabilities from one form to another to handle real life ambiguous situations [3]. It has been proven that TakagiSugeno fuzzy systems with affine terms can smoothly approximate any nonlinear functions to any specified accuracy within any compact set, which provides a theoretical foundation for using T-S fuzzy model to represent complex nonlinear system approximately [2]. 
The paper is organized in five sections. After the introduction in Section 1, Section 2 reviews the previous year work done making use of adaptive neuro fuzzy system. Section 3 discusses the architecture of ANFIS, the fuzzy reasoning mechanism, membership functions and fuzzy rule sets. It continues with discussions on the procedure of hybrid learning and fuzzy model validation, the error of observations for checking and training data sets. Section 5 presents the conclusions of the research, and the outcomes of TB Stage for various observations. The paper ends with a list of references.

\section{Literature Survey}

In this section, we will introduce some related works in the field of fuzzy logic. A detailed survey of fuzzy logic techniques is found during this section. There are many works in literature that explains design and implementation of medical experts system. There are diverse application areas in which ANN and FL have been effectively implemented whether in isolation or complementing each other's strengths. A combined neuro-fuzzy approach has seen enormous preferences recently from researchers working in different domains. A comprehensive study of existing work in assorted areas using soft computing methodologies specifically focusing on neural networks and fuzzy logic can be found in [4].

Jang in 1993 explained a computational technique to deal with non-linear and complex problems. This study makes use of fuzzy inference system implemented in the construction of adaptive networks. The proposed adaptive system can generate an inputoutput mapping based on human knowledge and predetermined input-output data pairs by means of the hybrid algorithm. The simulation studies suggest that system architecture can be utilized to model nonlinear functions, identify nonlinear components online in a control system and to predict a chaotic time series. Further, author compared system with artificial neural networks and preliminary tested [4].

S. Mitra, et al., in 2000 surveyed a comprehensive study of neuro-fuzzy rule generation algorithms for real-time applications. An extensive investigation shows the qualitative better results can be obtained using rule extraction and rule refinement. The proposed algorithms makes use of fuzzy sets and an aid in giving information in a more human comprehensible or natural form and can handle uncertainties at various levels. Models are the group on the basis of their level of neuro-fuzzy synthesis. The proposed methodology has an additional benefit in other soft other soft computing tools like genetic algorithms and rough sets. Based on fuzzy inference system, real-life application to medical diagnosis is provided [5].

The problem of inferring operating rules using an ordinary regression, an FR, and an adaptive neuro-fuzzy model was explored by S. J. Mousavi, et al., in 2006 for reservoir operations. Author used two types of models, a long-term planning and a medium-term implicit stochastic optimization to predict accuracy of system. Computational results shows that ANFIS was performed better and fitted to optimal input-output data set derived from a reservoir optimization model, the FR has shown to be superior in the long-term planning problem. Finally for future works, author recommended the methods examined during this study may be assessed in more complex multireservoir multipurpose systems. Moreover, they can be compared with the policies resulting from explicit stochastic models [11].

M. Huang, et al., in 2007 carried an integrated approach for glaucoma detection using adaptive neuro fuzzy inference system. The purpose of this study was to develop an automated classifier based on adaptive neuro fuzzy inference system, to differentiate between normal and glaucomatous eyes from the quantitative evaluation of summary data reports of the Stratus optical coherence tomography (OCT) in Taiwan Chinese population. In this work decision making was performed in two stages; features are selected using orthogonal array and then training is performed by using backpropagation gradient method in combination with least square method. It is investigated that using stratus OCT 
parameter as input, ANFIS generates better results for discriminating between glaucomatous and normal eyes. Therefore, use of adaptive neuro fuzzy inference system is preferable since output concludes the if-then rules and membership functions, which enhances the readability of the output [6].

A. Yardimci in 2009 surveyed on various soft computing techniques in medicinal field and aims to describe briefly about various soft computing methodologies between years 2000 and 2008. In this paper research work research work on MEDLINE was explained. Basic study involves the current elements of soft computing, different mechanisms implemented in soft computing techniques and to establish a guide which help to approximation the future developments of soft computing in medicine. Further, use of soft computing methods in wide range of fields such as imaging, classification, diagnosis, prediction and control in medicine was suggested [7].

Usefulness of adaptive neuro fuzzy inference for tuberculosis diagnosis was introduced by Tamer (2012). The proposed approach makes use of Sugeno-type adaptive-networkbased fuzzy inference system (ANFIS) to prognosticate the existence of mycobacterium tuberculosis. Dataset collected from 503 different patient records which were obtained from a private health clinic. The patient record has 30 different attributes which cover demographical and medical test data. ANFIS model was generated by using 250 records. The proposed model classifies the instances with the exactness of $97 \%$, whereas rough set algorithm does the same classification with an accuracy of $92 \%$. This learning has a contribution on forecasting patients before the medical tests [8].

N. Walia, et al., in 2015 proposed a decision support system for tuberculosis bacterium class identification. Using this framework, author suggested use of fuzzy diagnosability approach for diagnosis of mycobacterium tuberculosis and formalize reasoning. A fuzzy diagnosability function that takes values in the interval $(0,1)$ has been introduced to characterize the diagnosability degree using fuzzy systems. The proposed approach enables to deal with varying degree of input attributes, to solve problem of fuzziness, impreciseness and subjectivity during analysis process. Further, accuracy analysis of system was calculated and observed value is $77 \%$. The designed system helps medical pulmonary physicians during diagnosis of ailment by reducing time and to make prompt decision. With the results obtained, a further issue worthy of consideration to other chronic obstructive pulmonary diseases can be done. It was suggested that the designed system can be extended using number of inputs or using clustering techniques [3].

It is inferred from above literature survey on fuzzy logic and adaptive neuro system has been successfully applied in many medical fields for diagnosing and monitoring of various diseases.

\section{Clinical Decision Support System}

In real world computing environment, the information is not complete, precise and certain, making very intricate to derive a definite conclusion. Clinical decision support can be defined as active knowledge systems that form a significant part in field of clinical knowledge management technologies to generate case-specific advice. This system makes use of knowledge management in such a way to achieve clinical advice for patient care based on multiple items of patient data. Various Clinical Decision Support Systems have been constructed with the help of Artificial intelligence. Usages of these systems are now widely available in various hospitals and health clinics. They are proved to be very valuable for patient as well as for medical experts in making the prompt decisions about the ailment. Clinical decision support system is broadly classified into two main categories; (i) Knowledge-based clinical decision support system (ii) Non-knowledge based clinical decision support system [1] as shown in Figure 1. 


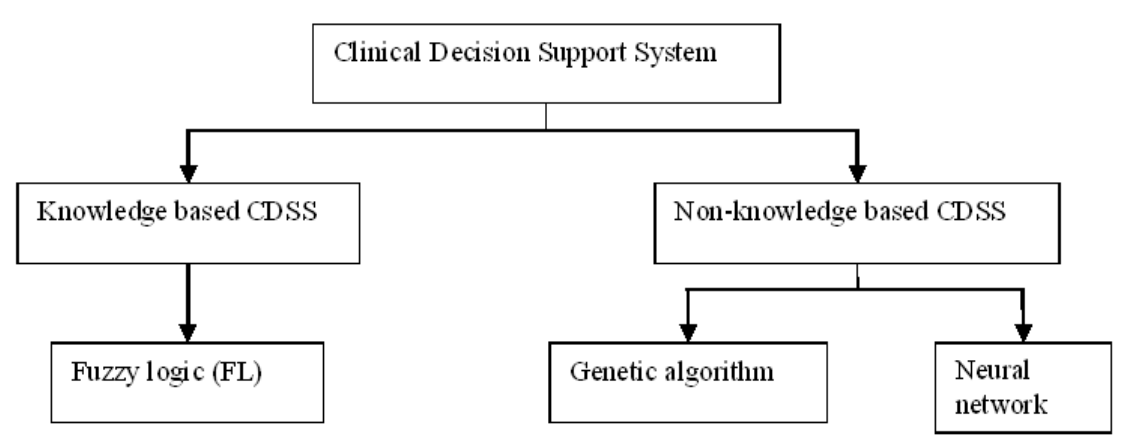

Figure 1. Clinical Decision Support System using Different Methodological Branches

The knowledge-based decision support system framework mainly includes usage of Ifthen type rules, which are also referred as production rules to conclude the output of reasoning mechanism. The input data is usually associated with these rules. For example, if fever intensity is high then generate warning severe. The knowledge-based system generally divided into three main parts: knowledge base, database rules and inference engine mechanism to compute effectively. Knowledge based system comprises of the database model and fuzzy logic model. The inference engine uses set of rules to combine patient information and to provide output. Fuzzy systems are useful in two general contexts: (i) situations involving highly complex systems whose behaviour are not well understood, and (ii) in situations where approximate, but fast solution is required [14, 3]. Such inference procedure derives conclusions from a set of fuzzy if-then rules and known facts. Fuzzy reasoning is also known as approximate reasoning. Fuzzy reasoning is made by using basic rules of inference such as two valued logic modus ponens in which the truth value of one proposition can be inferred from the truth value of other proposition given that one proposition implicates other. Fuzzy systems implement nonlinear systems making use of linguistic variables in a straightforward method when adequate knowledge about the system is available. The fuzzy logic module was used as a decision-making tool to resolve any uncertainty in the decision made by the neural networks. Fuzzy Set Theory (FST), a dominant tool used to handle imprecision and uncertainty can be used to deal with the concept of partial true and partial false values aiming attractability, stoutness and low cost solutions for real world challenges.

The system that focuses on the procedure having no knowledge base data is termed as machine learning algorithm or non-knowledge based clinical decision support system. It is further classified into two main categories as neural networks (NN), genetic algorithm (GA).

\subsection{Neural Network}

In order to attain relationship between the symptoms and diagnosis, neural networks make use of nodes and weighted connections. This fulfills the need not to write rules for input. However, the system fails to explain the reason for using the data in a particular way. Simulation studies shows that the self-organizing process of training the neural network in which it isn't given any priory information about the categories it is required to identify, is capable of extracting appropriate information from input data in order to produce clusters that correspond to class. Furthermore it requires only a small proportion of available data to train the network. Usage of neural networks is very important especially in complex multi-variable systems in order to avoid costly medical treatment. Neural Networks have been widely useful for modeling complex databases of medical information and to solve non-linear statistical modeling problems. Training of neural network-based systems can be done by using numerical data and fuzzy rules can be extracted from neural networks. These networks are trained in order to optimize 
performance of network in estimating output for particular input space. Back propagation training algorithm, a popular approach used with medical databases adjusts weight of an ANN to minimize a cost function. The ANN maintains correct classification rates and allows a large reduction in complexity of the systems. The use of the weight-elimination cost function is well enough to overcome the network memorization problems.

\subsection{Genetic Algorithm (GA)}

Genetic algorithm is subclass of an evolutionary algorithm that utilizes biology inspired mechanism, where elements of search space are binary strings (chromosomes) which correspond to a particular solution. GA is a powerful tool for optimization of fuzzy rule-based system and complex problems. Selection algorithm evaluates components of solutions to a problem. The generic system goes through an iterative procedure in order to achieve the best solution of a problem. These systems are deployed for optimal selection of antecedents and consequents in a fuzzy system. The major weakness of genetic systems is that it usually tends to be, (i) computationally expensive in real systems, (ii) premature convergence and (iii) slow search speed. This system is appropriate when the best solution is not required, only appropriate solution is required $[9,10]$. GAs are better than traditional search techniques as they donot need to biased to acquire the locally optimum solution. The robustness of the GA is due to their capacity to locate the global optimum in a large and complex scenario. Genetic learning processes comprises different levels of complexity based on different types of structural changes produced by the algorithm, from the simplest case of parameter optimization to the highest level of complexity of learning the rule set of a rule based system. GA has acquired immense consideration in solving engineering problems where large and multipart space exists, and has been used productively used in a wide range of applications such as function optimization [12]. GAs are better than traditional search techniques as they donot need to biased to acquire the locally optimum solution. The robustness of the GA is due to their capacity to locate the global optimum in a large and complex scenario.

\section{Adaptive Neuro Fuzzy Inference System}

Jang in 1993 proposed architecture and learning algorithms which is combination of fuzzy logic with neural networks for drawing inference [4]. It is a model that is proficient in constructing input-output mapping accurately based on both human knowledge using data in the form of fuzzy if-then rules and predetermined input output data pairs. The adaptive-network-based fuzzy inference system maps input data using input membership functions (MFs) with its associated parameters, and then through output MFs to conclude outputs. The initial membership functions and rules for the fuzzy inference system can be calculated by employing human expert knowledge about the target system to be modelled. ANFIS can then refine the fuzzy if-then rules and membership functions to illustrate the input-output behaviour of a complex system [13]. Multi-layer adaptive network-based fuzzy inference architecture consists of totally five layers to implement different node functions to learn and tune parameters in a FIS using a hybrid learning mode. The hybrid learning algorithm allows identifying parameters of Sugeno-type fuzzy inference systems. It applies a combination of the least-squares method and the back-propagation gradient descent method for training FIS membership function parameters to follow a given training data set. Figure 2 shows entire system architecture consists of five layers, namely fuzzy layer, product layer, normalized layer, de-fuzzy layer and output layer. Hybrid neuro system is a powerful framework for handling practical classification problems and is able to create class boundaries that reduce its misclassification rates.

During the forward pass, with fixed premise parameters, the least squared error estimation approach is employed to update the consequent parameters and to pass the errors to the backward pass. 


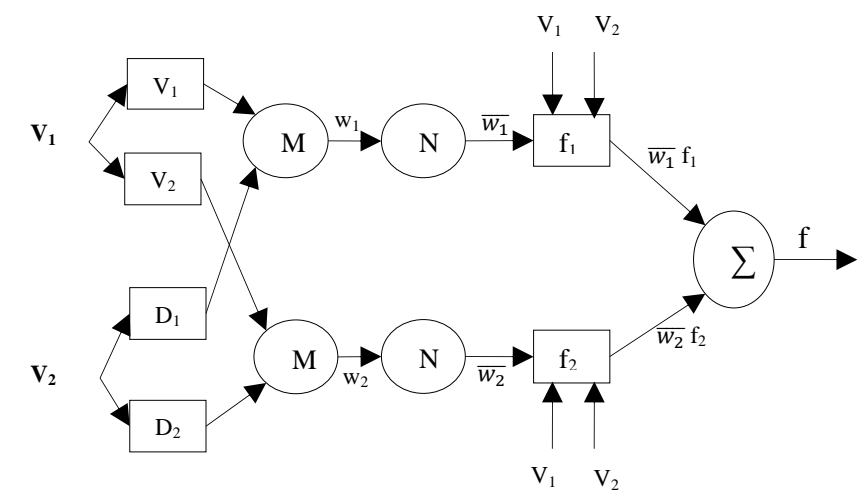

Figure 2. Basic ANFIS Structure for Two Input Sugeno Fuzzy Model

The first-order Sugeno fuzzy model allows use of consequent part of fuzzy inference system having linear values and the parameter can be predicted by using a simple leastsquares error method. For simplicity, it is assumed that FIS under consideration has two inputs, $\mathrm{x}$ and $\mathrm{y}$, and one output, $\mathrm{z}$. A typical rule set having two fuzzy if-then rules Takagi and Sugeno fuzzy rules can be expressed as using Equation 1.

$$
\begin{aligned}
& \text { Rule 1: If } x \text { is } A_{1} \text { and } y \text { is } B_{1} \text { then } z_{1}=p_{1} x+q_{1} y+r_{1} \\
& \text { Rule 2: If } x \text { is } A_{2} \text { and } y \text { is } B_{2} \text { then } z_{2}=p_{2} x+q_{2} y+r_{2}
\end{aligned}
$$

where $A_{1}, A_{2}$ and $B_{1}, B_{2}$ are the membership functions for the inputs $x$ and $y$, respectively; $\mathrm{p}_{1}, \mathrm{q}_{1}, \mathrm{r}_{1}$ and $\mathrm{p}_{2}, \mathrm{q}_{2}, \mathrm{r}_{2}$ are linear parameters having values in the then-part of fuzzy if-then rules, and are called consequent parameters. The architecture of ANFIS consists of five layers, and an introduction of the model layers is presented below.

Layers 1: Each input node $\mathrm{i}$ in this layer is an adaptive node which generates membership grade of linguistic variable. It is a fuzzy layer, in which $\mathrm{v}$ and $\mathrm{d}$ are input of system. $\mathrm{O}_{1, \mathrm{i}}$ is the output of the $\mathrm{i}^{\text {th }}$ node of layer 1 . Each adaptive node is a square node with square function defined by using Equation (2):

$$
\begin{gathered}
\mathrm{O}_{1, \mathrm{i}}=\mu_{\mathrm{v}, \mathrm{i}}(\mathrm{v}) \text { for } \mathrm{i}=1,2 \\
\mathrm{O}_{1, \mathrm{j}}=\mu_{\mathrm{d}, \mathrm{j}}(\mathrm{v}) \text { for } \mathrm{j}=1,2
\end{gathered}
$$

where $\mathrm{O}_{1, \mathrm{i}}$ and $\mathrm{O}_{1, \mathrm{j}}$ denote output function and $\mu_{\mathrm{v}, \mathrm{i}}$ and $\mu_{\mathrm{d}, \mathrm{j}}$ denote membership function. Both bell-shaped and gaussian membership function can be used for fuzzy sets due to their nonlinear, smooth and continuous derivatives. Parameters in this layer are referred to as 'premise parameter'.

Layer 2: Every node in this layer is fixed. This layer receives input values $\mathrm{v}_{\mathrm{i}}$ from first layer and acts as a membership function to represent fuzzy sets of respective input variables to check weights of each membership function. Each node in this layer estimates the firing strength $\left(\mathrm{w}_{\mathrm{i}}\right)$ of a rule. The output in this layer can be represented using Equation 3:

$$
\mathrm{O}_{2, \mathrm{i}}=\mathrm{w}_{\mathrm{i}}=\mu_{\mathrm{v}, \mathrm{i}}(\mathrm{v}) \cdot \mu_{\mathrm{Dj}}(\mathrm{d}), \mathrm{i}=1,2
$$

In general, any T-norm operator that performs fuzzy AND can be used as a node function in this layer.

Layer 3: Every node in this layer is a circle node labelled with $\mathrm{N}$, indicating normalization to the firing strength from previous layer. The ith node performs precondition matching of fuzzy rules, i.e. it calculates the ratio of the ith rule's firing strength to the sum of all rules firing strengths. The output of this layer can expressed as $\overline{\mathrm{w}_{\mathrm{i}}}$ using Equation 4.

$$
\mathrm{O}_{3, \mathrm{i}}=\overline{\mathrm{w}_{\mathrm{i}}}=\frac{\mathrm{w}_{\mathrm{i}}}{\mathrm{w}_{1}+\mathrm{w}_{2}}, \mathrm{i}=1,2
$$

For convenience, output of this layer is known as normalized firing strengths. 
Layer 4: In this layer, the nodes are adaptive nodes. The resultant output is simply a product of normalized firing rule strength and first order polynomial. Weighted output of rule represented by node function and computed as using equation 5:

$$
\mathrm{O}_{4, \mathrm{i}}=\overline{w_{i}} \mathrm{f}_{\mathrm{i}}=\overline{w_{i}}\left(\mathrm{p}_{\mathrm{i}} \mathrm{v}+\mathrm{q}_{\mathrm{i}} \mathrm{d}+\mathrm{r}_{\mathrm{i}}\right), \quad \mathrm{i}=1,2
$$

where $\mathrm{O}_{4, \mathrm{i}}$ represents layer 4 output. In this layer, $\mathrm{p}_{\mathrm{i}}, \mathrm{q}_{\mathrm{i}}$ and $\mathrm{r}_{\mathrm{i}}$ are linear parameter or consequent parameter and $\mathrm{fi}$ is a linear function of input variables.

Layer 5: This layer aggregates the qualified consequents to produce a crisp output. The single node in this layer is fixed which transforms fuzzy classification results into crisp values. It computes the weighted average of all incoming signals to calculate output signals calculated using equation 6 .

$$
\mathrm{O}_{5, \mathrm{i}}=\sum_{\mathrm{i}} \overline{\mathrm{w}_{\mathrm{i}}} \mathrm{f}_{\mathrm{i}}=\frac{\sum_{\mathrm{i}} \mathrm{w}_{\mathrm{i}} \mathrm{f}_{\mathrm{i}}}{\mathrm{w}_{1}+\mathrm{w}_{2}}, \quad \mathrm{i}=1,2
$$

Thus, it is observed that when the values of premise parameter are fixed, the overall output of the adaptive network can be expressed as linear combination of a consequent parameter.

Hybrid neuro fuzzy systems can be used in diagnosis of pulmonary lung diseases, cortical malformations, hepatitis and diabetes, rheumatic and pancreatic diseases. A fuzzy rule based expert system can be designed to detect stage of tuberculosis and accordingly these fuzzy rules are updated using rule mining techniques. Based on this technique that generates classes of tuberculosis suits the needs of pulmonary physicians and reduce the time consumed in generating diagnosis of ailment [14]. Fuzzy cognitive maps are used in the modeling of meningitis, as a support tool for physicians in the accurate diagnosis and treatment of the condition [1].

\section{Conclusion}

We have described the clinical decision support system along with architecture of adaptive network based fuzzy inference systems (ANFIS). Based on hybrid learning algorithm, the proposed architecture can improve the quality of generated relevant fuzzy if-then rules obtained from human experts to illustrate the input-output behavior of a complex system. Fuzzy logic provides a means for drawing the subjective decision making process in an algorithm suitable for computer implementation.

\section{Acknowledgments}

Navneet Walia author wishes to express her sincere gratitude to Harsukhpreet Singh, Assistant Professor, CT Institute of Technology and Research and Mr. Sharad Tiwari, Research Scholar, Thapar University, Patiala and anonymous reviewers for their invaluable suggestions that greatly help to improve the quality of paper.

\section{References}

[1] V. Prasath, N. Lakshmi, M. Nathiya, N. Bharathan and N. P. Neetha, "A Survey on the Applications of Fuzzy Logic in Medical Diagnosis support systems systems decision," International Journal of Scientific \& Engineering Research, ISSN 2229-5518, vol. 4, no. 4, (2013), pp. 1199-1203.

[2] N. Walia, H. Singh and A. Sharma, "ANFIS: Adaptive Neuro-Fuzzy Inference System- A Survey," International Journal of Computer Applications, vol. 123, no. 13, (2015), pp. 32-38.

[3] N. Walia, H. Singh, S. K. Tiwari and A. Sharma, "A Decision Support System for Tuberculosis Diagnosability," International Journal on Soft Computing (IJSC), vol. 6, no. 3, (2015), pp. 1-14.

[4] J. R. Jang, "ANFIS: Adaptive Network-Based Fuzzy Inference System,” IEEE Transactions on Systems, Man, and Cybernetics, vol. 23, no. 3, (1993).

[5] S. Mitra, Y. Hayashi and S. Member, "Neuro- Fuzzy Rule Generation: Survey in Soft computing framework," IEEE Transactions on Neural Networks, vol. 11, no. 3, (2000), pp. 748-768.

[6] M. Huang, "Glaucoma detection using adaptive neuro-fuzzy inference system," Expert Systems with Applications, Elsevier, doi:10.1016/j.eswa.2005.12.010, vol. 32, (2007), pp. 458-468. 
[7] A. Yardimci, "Applications of soft computing to medical problems," Ninth International Conference on Intelligent Systems Design and Applications, (2009), DOI: 10.1109/ISDA.2009.168.

[8] T. Uc, A. Karahoca and D. Karahoca, "Tuberculosis disease diagnosis by using adaptive neuro fuzzy inference system and rough sets," Neural Comput \& Applic, DOI: 10.1007/s00521-012-0942-1, (2013), pp. 471-483.

[9] R. Malhotra, N. Singh and Y. Singh, "Soft Computing Techniques for Process Control Applications," International Journal on Soft Computing ( IJSC ), vol. 2, no. 3, (2011), pp. 32-44.

[10] R. Malhotra, N. Singh and Y. Singh, "Genetic Algorithms: Concepts , Design for Optimization of Process Controllers," Computer and Information Science, vol. 4, no. 2, (2011), pp. 39-54.

[11] S. J. Mousavi, K. Ponnambalam and F. Karray, "Inferring operating rules for reservoir operations using fuzzy regression and ANFIS," Fuzzy Sets and Systems, Elsevier, doi:10.1016/j.fss.2006.10.024, vol. 158, (2007), pp. 1064-1082.

[12] M. Eftekhari and S. D. Katebi, "Extracting compact fuzzy rules for nonlinear system modeling using subtractive clustering, GA and unscented filter," Applied Mathematical Modelling, Elsevier, doi:10.1016/j.apm.2007.09.023, vol. 32, (2008), pp. 2634-2651.

[13] X. Lou and K. A. Loparo, "Bearing fault diagnosis based on wavelet transform and fuzzy inference," Mechanical Systems and Signal Processing, Elsevier, doi:10.1016/S0888-3270(03)00077-3, vol. 18, (2004), pp. 1077-1095.

[14] N. Walia, S. K. Tiwari and R. Malhotra, "Design and Identification of Tuberculosis using Fuzzy Based Decision Support System," Advances in Computer Science and Information Technology (ACSIT), ISSN: 2393-9907, vol. 2, no. 8, (2015), pp. 57-62.

\section{Author}

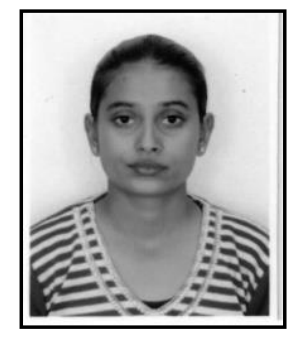

Navneet Walia, is pursuing M.TECH final year in department Electronics and Communication Engineering at CT Institute of Technology and Research, Jalandhar. She has done her B.TECH in trade Electronics and Communication engineering from CEM college of Engineering and Management. She has presented many papers in national and international conferences. Her topic of research is fuzzy logic, neuro-fuzzy, genetic algorithm and its applicability to industrial sector. 